\title{
The Role of Hydrogen Bonding in the Dehydration of Bioalcohols in Hydrophobic Pervaporation Membranes
}

\author{
Rafael M. Madero-Castro ${ }^{1,2}$, Sofia Calero*2,3 and A. Ozgur Yazaydin*1
}

${ }^{1}$ Department of Chemical Engineering, University College London, London, WC1E 7JE, UK

${ }^{2}$ Department of Physical, Chemical and Natural Systems, Universidad Pablo de Olavide, Ctra. Utrera Km. 1 Seville ES-41013, Spain

${ }^{3}$ Materials Simulation and Modelling, Department of Applied Physics, Eindhoven University of Technology, 5600 MB Eindhoven, The Netherlands.

Corresponding authors:

Email: ozgur.yazaydin@ucl.ac.uk

Email: scalero@upo.es

\begin{abstract}
The dehydration of bioalcohols is considered one of the major factors contributing to the cost of biofuel production. In this study, liquid phase separation of water from methanol and ethanol in a siliceous MFI pervaporation membrane was studied by performing concentration gradient driven molecular dynamic (CGD-MD) simulations. CGD-MD simulations work by imposing a higher concentration in the feed side and a lower concentration in the permeate side of the membrane. This creates a concentration gradient across the membrane that facilitates the diffusion of molecules from the feed to the permeate side, mimicking the experimental pervaporation membrane set up. Fluxes of methanol, ethanol and water were calculated in single component permeation simulations and in equimolar methanol-water and ethanol-water mixture separation simulations. It was found that water formed hydrogen bonding with the silanol ( $\mathrm{Si}-\mathrm{OH})$ groups on the external surface of the MFI and did not enter the membrane in the single component permeation simulation. While this may suggest that MFI can be used to effectively dehydrate bioalcohols, our simulations showed that water permeated through the MFI membrane when it was in a mixture with either methanol or ethanol. Furthermore, in the alcohol-water mixture simulations, the fluxes of methanol and ethanol were significantly lower than that of expected based on their single component fluxes. A detailed analysis of hydrogen bonding in the alcohol-water mixture separation simulations revealed that water preferred making hydrogen bonds with methanol and ethanol rather than with the silanol groups. This resulted in drifting of water molecules along with permeating alcohol molecules in to the MFI membrane in mixture simulations, while slowing the permeation of methanol and ethanol fluxes.
\end{abstract}

KEYWORDS: dehydration • bioalcohol • water • hydrogen bonds $\bullet$ separation 


\section{Introduction}

The warming of our planet has been closely linked to the anthropogenic release of greenhouse gases to the atmosphere.[1] To prevent further increase of Earth's temperature, substitution of fossil fuel based energy with that of generated from renewable resources, such as solar, $[2,3]$ wind $[4,5]$ and biomass, $[6$, 7] has been considered essential. However, the effectiveness of these alternative energy resources in the fight against climate change has not been without controversy.[8] In the case of biofuels in particular, there has been an ongoing debate about how environmentally friendly they are in reality.[9] For instance, some studies suggest that production of biofuels consume more energy than they produce.[10, 11] One of the major factors contributing to the cost of biofuel production is the dehydration of bioalcohols. This process has traditionally employed distillation, which requires large amounts of energy to achieve sufficient purity for alcohol to be used as fuel. Furthermore, ethanol, for instance, makes an azeotrope with water. Therefore it is not possible to obtain more than 95 wt \% purity ethanol with distillation only. Such difficulties have led to the development of alternative methods for dehydration of bioalcohols, such as, extractive distillation, adsorption and membrane separation, as well as, hybrid methods which incorporate membrane separation and distillation.[12-14]

Membrane pervaporation has been considered as one of the most effective and energy-saving processes for separation of alcohol-water mixtures, in particular, for the azeotropic ones.[15-17] Hydrophobic and hydrophilic membranes have been developed for the separation of alcohol and water,[12] although making reliable and economical membranes is challenging.[18] For the fabrication of hydrophobic pervaporation membranes, MFI, a hydrophobic zeolite, has been widely used, either in the form of a thin film membrane deposited on a porous support, [19, 20] or by dispersing MFI crystals in a polymer matrix,[21, 22] exhibiting high flux and separation factors. Furthermore, several molecular simulation studies investigated the adsorption, diffusion and separation of alcohol and water systems in MFI zeolite.[23-26]

By carrying out configurational bias grand canonical Monte Carlo (CB-GCMC) simulations Xiong et al.[23] reported that the adsorption of water in MFI is negligible due to its hydrophobicity and that larger alcohols are adsorbed at relatively lower pressures compared to the smaller alcohols. Krishna et al.[24] employed CB-GCMC and molecular dynamics (MD) methods to show that it is not possible to accurately estimate adsorption and diffusion characteristics of alcohol-water mixture based on single component data due to the effect of hydrogen bonding between water and alcohol molecules. GómezÁlvarez et al.[25] performed GCMC and MD simulations in MFI and showed that the diffusion coefficients of water, methanol and ethanol in the adsorbed alcohol-water mixtures were greater than their diffusion coefficients in the adsorbed single component. Studies cited in references 23 to 25 were conducted in periodic MFI structures. Jia et al.,[26] on the other hand, carried out MD simulations which involved the diffusion of alcohol-water mixtures through MFI slabs that mimicked pervaporation separation experiments. They emphasized that the behaviour of mixtures differ considerably from that of for the single component systems.

In this work, we investigate the dehydration of bioalcohols in an MFI membrane by carrying out concentration gradient driven molecular dynamics simulations (CGD-MD).[27] CGD-MD is a non- 
equilibrium molecular dynamics simulation method which works by applying bidirectional bias forces to maintain the concentration of molecules in designated control volumes. By maintaining a higher concentration at the inlet of a membrane (feed), and lower concentration at the outlet of a membrane (permeate) the CGD-MD method creates a concentration gradient which drives the diffusion of molecules through the membrane. This method was previously used to study multicomponent gas transport and separation in porous membranes.[27-29] Using the CGD-MD approach allows computing the steady-state flux of molecules and membrane selectivity directly, which is important, because some of us previously showed that at conditions where fluid molecules strongly associate, selectivity of a membrane may not always be computed accurately based on predictions of single component transport properties.[28] Here, we consider the separation of methanol and ethanol from water in an MFI pervaporation membrane setting; i.e., vacuum on the permeate side. The simulations are carried out in the liquid phase and at ambient conditions, and thus useful to understand the potential of porous membranes for the dehydration of bioalcohols with minimum energy consumption; i.e. without heating of the feed. Most importantly, our study reveals that water molecules prefers forming hydrogen bonds with alcohol molecules over the silanol groups that exist on the external surfaces of the MFI membrane. This leads to a diminishing effect on the alcohol-water selectivity and lower than expected alcohol fluxes.

\section{Simulation Methods}

Methanol and ethanol molecules were modelled with the TraPPE-UA force field.[30] In this force field, bonds are rigid but angles are flexible, and $\mathrm{CH}_{\mathrm{x}}$ groups are treated as united atoms, i.e., single interaction centres. For the water molecule, a flexible variant of the SPC/E model was used.[31, 32] An MFI membrane was constructed by using the "Computation-ready 2D Zeolitic Slabs Database" of Knio et al. $^{36}$ This database consists of DFT optimized unit cells of zeolites and their model surfaces for different crystallographic planes terminated with $-\mathrm{OH}$ groups. The dimensions of the MFI membrane used in our simulations were $60.49,53.17$ and $110.83 \AA$ in the $\mathrm{x}, \mathrm{y}$ and z direction, respectively, and the external surfaces were perpendicular to the straight channels [010]. The membrane was treated as a flexible structure using the force field developed by Sastre et al.[33] Lorentz-Berthelot mixing rules were used to calculate the cross term parameters between different atom types for Lennard-Jones (LJ) potential, which was used to model short range non-bonded interactions. Long range electrostatic interactions were computed using the Ewald summation method.[34] The cut-off distance for the LJ potential and the real part of the Ewald sum was set to $12 \AA$. Further details on the force field used and all parameters are provided in Table $\mathrm{S} 1$.

As mentioned in the introduction, we used the CGD-MD method to study the liquid phase transport of water, methanol and ethanol, and the separation of equimolar methanol-water and ethanol-water mixtures. The CGD-MD simulation setup is illustrated in Figure 1 and CGD-MD specific parameters are provided in Table S2. A detailed explanation of how CGD-MD method works can be found in Ozcan et al.[27] and Perego et al.[35] In our CGD-MD simulations, the MFI membrane was placed in the centre of the simulation box, which was $311.0 \AA$ in the $\mathrm{z}$ direction (Figure 1). During the CGD-MD simulations, the concentration of the water, methanol and ethanol molecules were maintained at their 
target densities in the feed control region (FCR) and the permeate control region (PCR), to mimic a pervaporation membrane separation setup; i.e. higher concentration in FCR (feed) and vacuum in PCR (permeate). This is achieved by applying bi-directional bias forces on fluid molecules in the feed force region (FFR) and the permeate force region (PFR). The bias force works in such a way that if the instantaneous concentration in the control region is less than the target concentration then bias force acts in the direction to move more molecules to the control region; and if the instantaneous concentration in the control region is more than the target concentration then the bias force acts in the direction to remove molecules from the control region. We emphasize that it is the concentration gradient that is established across the membrane which facilitates the transport of the molecules, not the bias forces. Molecules which cross the membrane return to the feed side through the periodic boundary (see blue arrows in Figure1). This ensures that there are always molecules in the feed side and that the transport of the molecules through the membrane can reach to steady state.

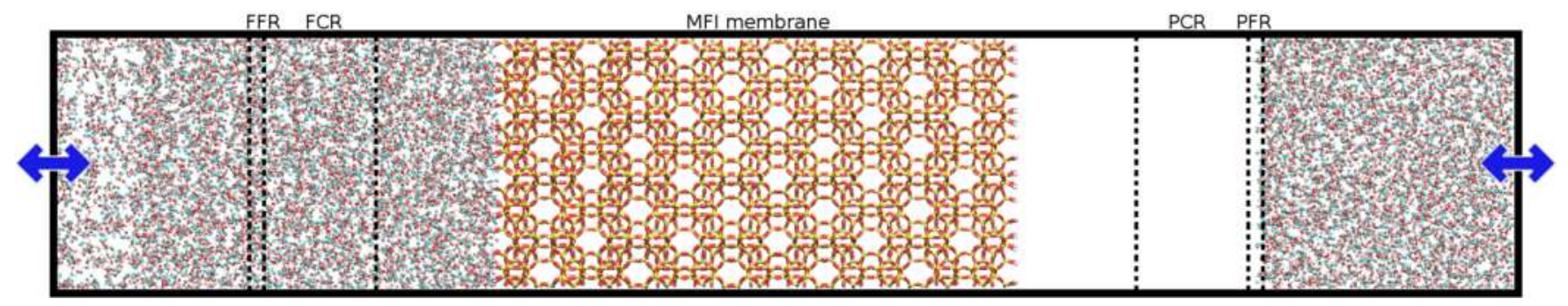

Figure 1. CGD-MD simulation setup.

All CGD-MD simulations were carried out using the Gromacs MD simulation package (version 2019.2) patched with an in-house modified version of Plumed 2.4.2. The patch is freely available for download.[36] Simulations were run in the NVT ensemble and the temperature was kept at $300 \mathrm{~K}$ using a Nose-Hoover thermostat. Leapfrog algorithm[37] was used for integrating the Newton's equations of motion with a timestep of $1 \mathrm{fs}$. A small number of Si atoms (less than 2\%) were restrained to their initial positions in order to avoid the MFI membrane drifting under the concentration gradient. To ensure that the flux of the molecules through the MFI membrane has achieved steady state, CGD-MD simulations were first run for 200 million steps, followed by 200 million steps of productions runs, during which fluxes, z-density profiles and hydrogen bonding characteristics of water, methanol and ethanol were calculated in single component and mixture settings.

\section{Results \& Discussion}

\subsection{Force field validation}

We first validated the force field by computing the adsorption isotherms of methanol, ethanol and water in MFI at $300 \mathrm{~K}$ and comparing them against experimental data. For this purpose, we performed grand canonical Monte Carlo (GCMC) simulation using the RASPA molecular simulation package.[38] Further details of the GCMC simulations are provided in the SI. Figure 2 shows the comparison between simulated and experimental adsorption isotherms of methanol, ethanol and water in MFI.[23, 39] Simulated isotherms reproduce the fact that MFI adsorbs methanol and ethanol at a lower pressure 
compared to

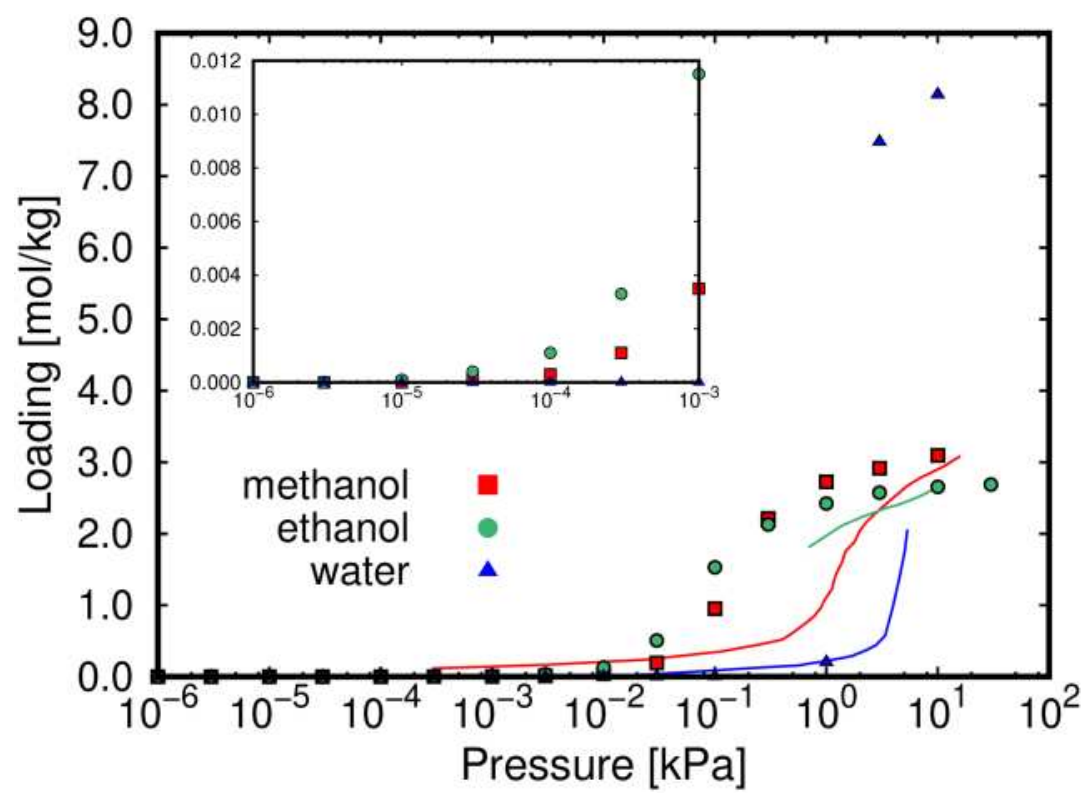

Figure 2. Comparison of the experimental (solid lines) and simulated (symbols) adsorption isotherms of methanol, ethanol and water in MFI.

water. It should also be noted that the amount of water adsorbed within the pressure range investigated in the GCMC simulations is much lower compared to the amount of water that condenses in the pores of defect free all-silica MFI at very high pressures; i.e. > $75 \mathrm{MPa} .[40,41]$

\subsection{Single component permeation of methanol, ethanol and water in the MFI membrane}

After validating the force field, we studied the single component permeation of methanol, ethanol and water in the MFI membrane by carrying out CGD-MD simulations. Target concentrations of methanol, ethanol and water in the feed control region were set to their liquid molar densities at $300 \mathrm{~K}$. in the permeate control region, the target concentrations were set to vacuum to mimic pervaporation conditions (Table S3). Instantaneous concentrations (Figure S1) and the average concentrations of methanol, ethanol and water (Table S3) in the control regions in single component simulations show that the concentration of the molecules were maintained very close to the target values during the production runs.

Figure 3 shows the z-density profiles of methanol, ethanol and water in the MFI membrane. While both methanol and ethanol enter the MFI membrane, water does not enter because of the hydrophobicity of the MFI zeolite. The z-density profiles of methanol and ethanol reflect the structure of MFI, i.e., the z- 
densities peak at the intersection of the zig-zag and straight channels.

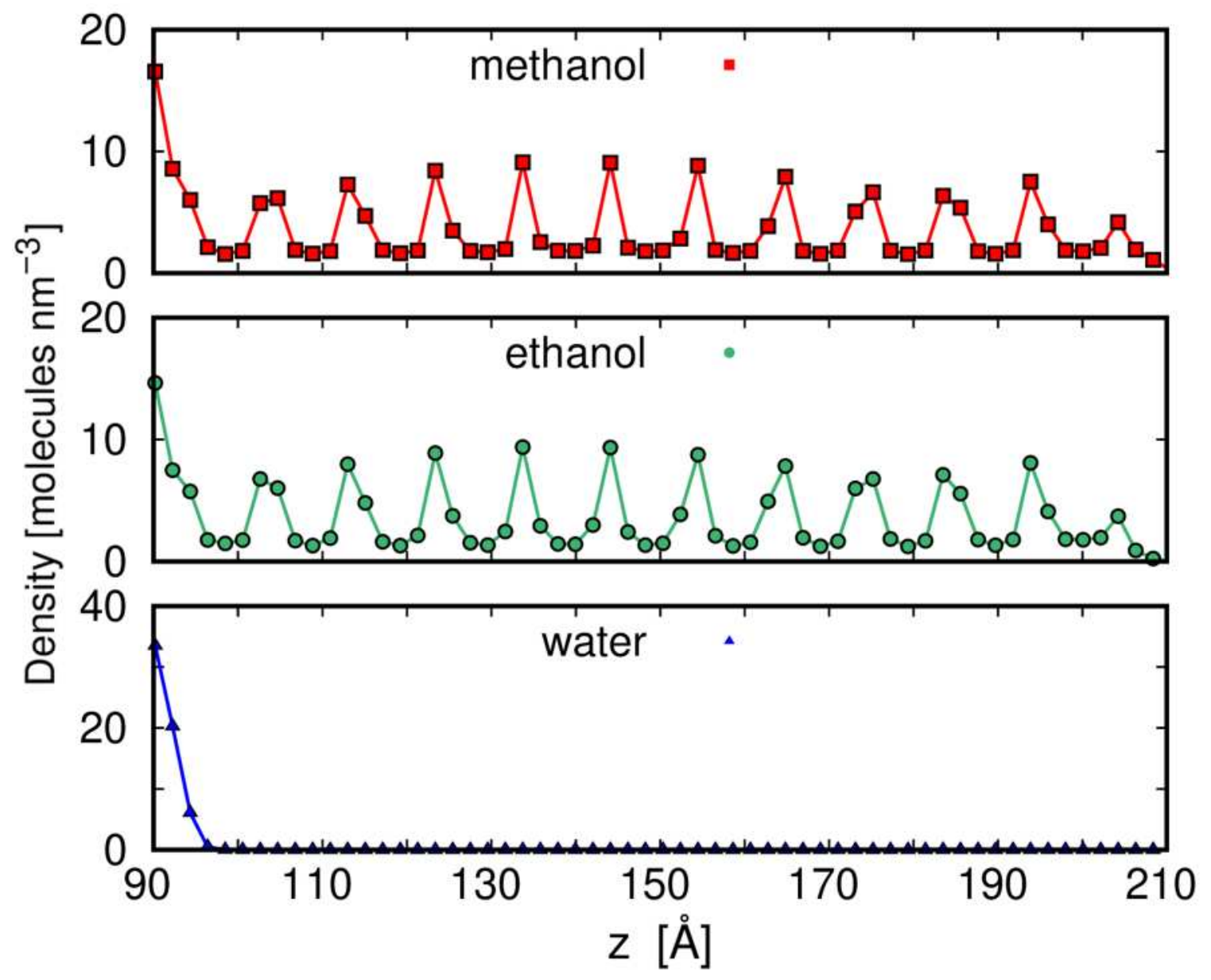

Figure 3. Z-density profiles of a) methanol, b) ethanol and c) water in the MFI membrane in single component CGD-MD simulations. The MFI membrane is located between $\mathrm{z} \approx 93 \AA$ and $204 \AA$.

The fluxes of methanol, ethanol and water in the MFI membrane were calculated by using the following formula[27]

$$
J_{z}=\frac{N_{i}^{+}-N_{i}^{-}}{t A_{x y}}
$$

where $J_{Z}$ is the flux in the direction of the flow, $N^{+}$and $N^{-}$are the number of molecules that cross a geometric plane located at the centre of the membrane in the positive and negative z-directions, respectively, $A_{x y}$ is the cross sectional area of the membrane, and $t$ is the simulation time. In the single component simulations, methanol flux is about twice that of ethanol, and water has a zero flux (Table 1) since it does not enter the MFI membrane, which is in agreement with the experimental findings that water only enters all-silica MFI at very high pressures.[40, 41] This may suggest that MFI can demonstrate complete selectivity for methanol and ethanol over water; however, as we show in the mixture simulations below this is not the case. 
Table 1. Methanol, ethanol and water fluxes, expressed in molecules $/ \mathrm{nm}^{2} \mathrm{~ns}$, in MFI from single component and mixture simulations.

\begin{tabular}{|c|c|c|c|}
\hline & Methanol & Ethanol & Water \\
\hline Single component & $0.20 \pm 0.01$ & $0.09 \pm 0.02$ & $0 *$ \\
\hline Methanol-water mixture & $0.013 \pm 0.008$ & - & $0.002 \pm 0.004$ \\
\hline Ethanol-water mixture & - & $0.03 \pm 0.01$ & $0.0005 \pm 0.0004$ \\
\hline
\end{tabular}

* Water does not enter the MFI membrane in the single component simulation.

\subsection{Dehydration of binary alcohol-water mixtures in the MFI membrane}

To study the dehydration of binary alcohol-water mixtures in the MFI membrane, we considered equimolar mixtures of methanol-water and ethanol-water. For both mixtures, target concentrations of the components in feed control region were set to reproduce the molar density of equimolar mixtures at 300 $\mathrm{K}$ assuming ideal mixing, and the target concentrations were set to zero in the permeate control region to mimic pervaporation conditions (Table S4). Instantaneous concentrations (Figure S2) and the average concentrations of methanol, ethanol and water (Table S4) in the control regions in mixture simulations show that the concentration of the molecules were maintained very close to the target values during the production runs.

Figure 4 shows the z-density profiles of methanol, ethanol and water in the MFI membrane in the mixture simulations. Unlike the case in the single component simulation of water, the density of water within the membrane, although very small, is non-zero in both mixture simulations. That is, water enters the membrane when it is in a mixture with methanol or ethanol. Indeed, there is a quantifiable water flux in MFI in the mixture simulations, although it is very small compared to the fluxes of methanol and ethanol (Table 1). Furthermore, the presence of water appears to be slowing down the permeation of methanol and ethanol in alcohol-water mixtures (Table 1). One can argue that the slower fluxes of methanol and ethanol in alcohol-water mixtures compared to their fluxes in the single component simulations is due to their relatively lower feed concentrations in the mixture simulation compared to their feed concentrations in the single component simulations. However, the lower feed concentrations of alcohols in the mixture simulations do not alone account for the slower fluxes of methanol and ethanol in the mixture simulations. For instance, the feed concentration of methanol in the mixture simulations is about $45 \%$ lower than that of its feed concentration in the single component simulation; i.e. 10.43 vs 14.99 [molecules $/ \mathrm{nm}^{3}$ ], respectively (Tables S3 and S4). The flux of methanol in the mixture simulation is about an order of magnitude smaller than its single component flux (Table 1). Likewise, the flux of ethanol in the mixture is three times smaller than its single component flux (Table 1 ), but the ethanol feed concentration in the mixture simulation is only $\approx 30 \%$ lower than that of in the single component; i.e. 7.92 vs 10.16 [molecules $/ \mathrm{nm}^{3}$ ], respectively (Tables S3 and S4). 


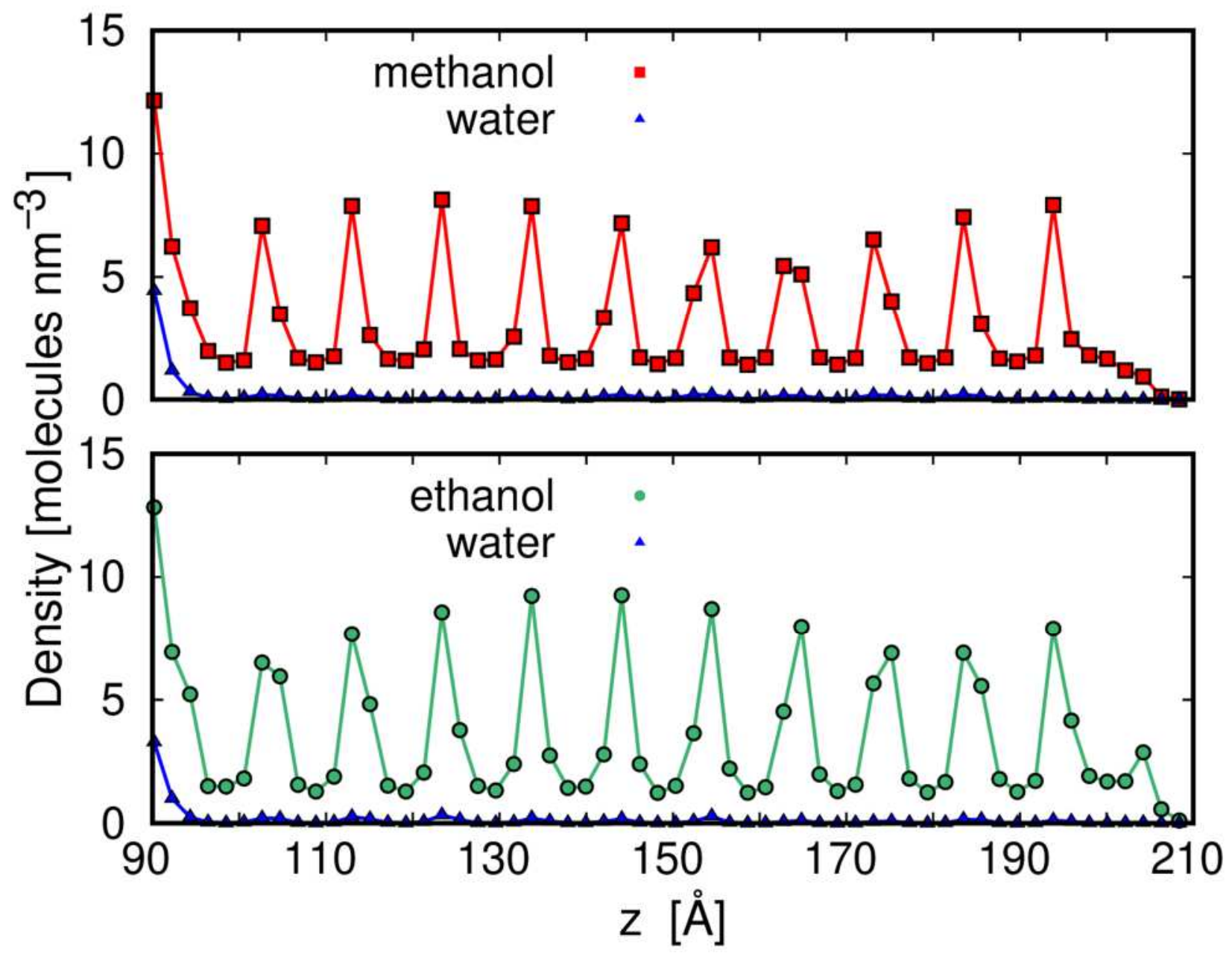

Figure 4. Z-density profiles of methanol, ethanol and water as a function of $\mathrm{z}$ coordinate in mixture CGD-MD simulations. The MFI membrane is located between $\mathrm{z} \approx 93 \AA$ and $204 \AA$.

To understand why water enters the MFI membrane in the alcohol-water mixture simulations but not in the single component water simulation, we analysed the number of hydrogen bonds that form between the silanol groups located on the feed side of the MFI surface and the methanol, ethanol and water molecules (Table 2). In single component simulations water molecules form about five times more hydrogen bonds with the surface silanol groups compared to methanol and ethanol. This may be due to two reasons; first, water has two - $\mathrm{OH}$ groups whereas methanol and ethanol has only one, and second, the molecular density of water is larger compared to methanol and ethanol, that is, there are relatively more water molecules present per unit volume in comparison to methanol and ethanol. However, in the mixture simulations there is a very different scenario. The number of hydrogen bonds that methanol or ethanol form with the silanol groups are more than the number of hydrogen bonds that form between the water molecules and silanol groups. The number of hydrogen bonds that water forms with silanol groups when it is in a mixture with methanol is about 10 times smaller compared to that of in the single component simulation, and it is 14 times smaller when it is in a mixture with ethanol. This cannot be 
explained only by the lower concentration of water in the mixture simulations (10.39 and 7.88 molecules $/ \mathrm{nm}^{3}$ for methanol and ethanol mixtures, respectively (Table S4)), compared to its concentration in the single component simulation, (33.7 molecules $/ \mathrm{nm}^{3}$ (Table S3)). It is clear that, water molecules interact more strongly with the alcohol molecules; i.e. through hydrogen bonding, compared to the surface silanols. This in turn facilitates the diffusion of water in the MFI membrane, because thanks to the hydrogen bonding that forms between the water and alcohol molecules, water molecules are carried in to the MFI with the diffusing methanol and ethanol molecules.[42] The preferred hydrogen bonding of water with alcohol molecules also explains the lower than expected fluxes of methanol and ethanol in their mixtures with water. While water enters MFI thanks to the hydrogen bonds that it forms with alcohol molecules, it slows down the permeation of methanol and ethanol.

Table 2. Number of hydrogen bonds that methanol, ethanol and water molecules form with the MFI surface at the feed side in single component and mixture simulations.

\begin{tabular}{|l|c|c|c|}
\hline & Methanol & Ethanol & Water \\
\hline Single component & $17 \pm 4$ & $17 \pm 3$ & $76 \pm 7$ \\
\hline Methanol-water mixture & $12 \pm 3$ & - & $8 \pm 3$ \\
\hline Ethanol-water mixture & - & $9 \pm 3$ & $5 \pm 2$ \\
\hline
\end{tabular}

The reason for the preference of water molecules for making hydrogen bonding with alcohols rather than with surface silanols can be due to the difference in the polarity of - $\mathrm{OH}$ groups in the silanol groups and those of in the methanol and ethanol molecules. Partial charges of oxygen atoms in the --OH groups of alcohol and the silanol are very similar (i.e., $q_{o_{a l c}}=-0.7 e$ and $q_{o_{\text {sil }}}=-0.725 e$ ). However, the partial charge of the hydrogen atom of the $-\mathrm{OH}$ group of alcohol is more than twice that of for the hydrogen atom of the $-\mathrm{OH}$ group of the silanol (i.e.; $q_{H_{a l c}}=0.435 e$ and $q_{H_{s i l}}=0.2 e$, respectively). The larger hydrogen partial charge on the alcohol $-\mathrm{OH}$ group leads to a stronger interaction with water compared to the interaction between the silanol group and water. This suggest that even if defect free membranes can be manufactured, the hydrogen bonding between water and alcohol molecules will always result in diffusion of water molecules along with alcohols, thus eliminating the possibility of complete dehydration of biofuels. Nevertheless, the ethanol/water selectivity based on the ethanol and water fluxes from the ethanol-water mixture simulation, i.e. $0.03 / 0.0005=60$, can still be considered very high.

\section{Conclusions}

The purification of bioalcohols using pervaporation membranes based on hydrophobic zeolites potentially has several advantages in comparison to distillation, including, most importantly, lower energy requirements. All silica MFI zeolite allows the diffusion of pure methanol and ethanol and 
prevents the diffusion of pure water due to its hydrophobic character. Pure water strongly hydrogen bonds with the silanol groups on the external surface of the MFI. On the other hand, when water is in a mixture with alcohol, the hydrogen bonding of water with silanol groups reduces due to its preferred hydrogen bonding with alcohols and this leads to water permeation through the MFI membrane along with alcohol molecules. While this may prohibit total selectivity of alcohol over water in a hydrophobic pervaporation membrane, such as MFI, it also hints at the importance of surface functional groups which may be modified to retain water outside the membrane. Our findings can aid in developing new strategies for manufacturing membranes with improved separation performance for the dehydration of bioalcohols.

\section{Acknowledgements}

The research leading to these results has received funding from Spanish Ministerio de Ciencia e Innovación (PID2019-111189GB-I00 and CTQ2017-92173-EXP). We are grateful to the UK Materials and Molecular Modelling Hub for computational resources, which is partially funded by EPSRC (EP/P020194/1 and EP/T022213/1).

\section{References}

[1] D. Lüthi, M. Le Floch, B. Bereiter, T. Blunier, J.M. Barnola, U. Siegenthaler, D. Raynaud, J. Jouzel, H. Fischer, K. Kawamura, T.F. Stocker, High-resolution carbon dioxide concentration record 650,000800,000 years before present, Nature, 453 (2008) 379-382.

[2] N.S. Lewis, D.G. Nocera, Powering the planet: Chemical challenges in solar energy utilization, Proceedings of the National Academy of Sciences, 103 (2006) 15729-15735.

[3] N.S. Lewis, Toward Cost-Effective Solar Energy Use, Science, 315 (2007) 798-801.

[4] R. Saidur, N.A. Rahim, M.R. Islam, K.H. Solangi, Environmental impact of wind energy, Renewable and Sustainable Energy Reviews, 15 (2011) 2423-2430.

[5] G.M. Joselin Herbert, S. Iniyan, E. Sreevalsan, S. Rajapandian, A review of wind energy technologies, Renewable and Sustainable Energy Reviews, 11 (2007) 1117-1145.

[6] P.S. Nigam, A. Singh, Production of liquid biofuels from renewable resources, Progress in Energy and Combustion Science, 37 (2011) 52-68.

[7] C. Somerville, H. Youngs, C. Taylor, S.C. Davis, S.P. Long, Feedstocks for Lignocellulosic Biofuels, Science, 329 (2010) 790-792.

[8] M.Z. Jacobson, Review of solutions to global warming, air pollution, and energy security, Energy \& Environmental Science, 2 (2009) 148-173.

[9] J.P.W. Scharlemann, W.F. Laurance, How Green Are Biofuels?, Science, 319 (2008) 43-44.

[10] S. Ulgiati, A Comprehensive Energy and Economic Assessment of Biofuels: When "Green" Is Not Enough, Critical Reviews in Plant Sciences, 20 (2001) 71-106.

[11] D. Pimentel, T. Patzek, G. Cecil, Ethanol production: energy, economic, and environmental losses, in: Reviews of environmental contamination and toxicology, Springer, 2007, pp. 25-41.

[12] H.-J. Huang, S. Ramaswamy, U.W. Tschirner, B.V. Ramarao, A review of separation technologies in current and future biorefineries, Separation and Purification Technology, 62 (2008) 1-21.

[13] A.K. Frolkova, V.M. Raeva, Bioethanol dehydration: State of the art, Theoretical Foundations of Chemical Engineering, 44 (2010) 545-556. 
[14] B. Claessens, J. Cousin-Saint-Remi, J.F.M. Denayer, Efficient Downstream Processing of Renewable Alcohols Using Zeolite Adsorbents, in, Springer Berlin Heidelberg, Berlin, Heidelberg, pp. $1-35$.

[15] M.H.V. Mulder, J.O. Hendrikman, H. Hegeman, C.A. Smolders, Ethanol-water separation by pervaporation, J. Membrane Sci., 16 (1983) 269-284.

[16] Y.K. Ong, G.M. Shi, N.L. Le, Y.P. Tang, J. Zuo, S.P. Nunes, T.-S. Chung, Recent membrane development for pervaporation processes, Progress in Polymer Science, 57 (2016) 1-31.

[17] P. Wei, L.-H. Cheng, L. Zhang, X.-H. Xu, H.-l. Chen, C.-j. Gao, A review of membrane technology for bioethanol production, Renewable and Sustainable Energy Reviews, 30 (2014) 388-400.

[18] Y. Huang, R.W. Baker, L.M. Vane, Low-Energy Distillation-Membrane Separation Process, Ind Eng Chem Res, 49 (2010) 3760-3768.

[19] X. Shu, X. Wang, Q. Kong, X. Gu, N. Xu, High-Flux MFI Zeolite Membrane Supported on YSZ Hollow Fiber for Separation of Ethanol/Water, Ind Eng Chem Res, 51 (2012) 12073-12080.

[20] D. Korelskiy, T. Leppäjärvi, H. Zhou, M. Grahn, J. Tanskanen, J. Hedlund, High flux MFI membranes for pervaporation, Journal of Membrane Science, 427 (2013) 381-389.

[21] F.S. Kamelian, T. Mohammadi, F. Naeimpoor, Fast, facile and scalable fabrication of novel microporous silicalite-1/PDMS mixed matrix membranes for efficient ethanol separation by pervaporation, Separation and Purification Technology, 229 (2019) 115820.

[22] T.C. Bowen, R.G. Meier, L.M. Vane, Stability of MFI zeolite-filled PDMS membranes during pervaporative ethanol recovery from aqueous mixtures containing acetic acid, Journal of Membrane Science, 298 (2007) 117-125.

[23] R. Xiong, S.I. Sandler, D.G. Vlachos, Molecular Screening of Alcohol and Polyol Adsorption onto MFI-Type Zeolites, Langmuir, 28 (2012) 4491-4499.

[24] R. Krishna, J.M. van Baten, Hydrogen Bonding Effects in Adsorption of Water-Alcohol Mixtures in Zeolites and the Consequences for the Characteristics of the Maxwell-Stefan Diffusivities, Langmuir, 26 (2010) 10854-10867.

[25] P. Gómez-Álvarez, E.G. Noya, E. Lomba, S. Valencia, J. Pires, Study of Short-Chain Alcohol and Alcohol-Water Adsorption in MEL and MFI Zeolites, Langmuir, 34 (2018) 12739-12750.

[26] W. Jia, S. Murad, Molecular dynamics simulation of pervaporation in zeolite membranes, Molecular Physics, 104 (2006) 3033-3043.

[27] A. Ozcan, C. Perego, M. Salvalaglio, M. Parrinello, O. Yazaydin, Concentration gradient driven molecular dynamics: A new method for simulations of membrane permeation and separation, Chemical Science, 8 (2017) 3858-3865.

[28] S. Namsani, A. Ozcan, A.Ö. Yazaydın, Direct Simulation of Ternary Mixture Separation in a ZIF-8 Membrane at Molecular Scale, Advanced Theory and Simulations, 2 (2019) 1900120.

[29] A. Ozcan, R. Semino, G. Maurin, A.O. Yazaydin, Modeling of Gas Transport through Polymer/MOF Interfaces: A Microsecond-Scale Concentration Gradient-Driven Molecular Dynamics Study, Chemistry of Materials, 32 (2020) 1288-1296.

[30] B. Chen, J.J. Potoff, J.I. Siepmann, Monte Carlo Calculations for Alcohols and Their Mixtures with Alkanes. Transferable Potentials for Phase Equilibria. 5. United-Atom Description of Primary, Secondary, and Tertiary Alcohols, The Journal of Physical Chemistry B, 105 (2001) 3093-3104.

[31] P. Mark, L. Nilsson, Structure and dynamics of the TIP3P, SPC, and SPC/E water models at 298 K, Journal of Physical Chemistry A, 105 (2001) 9954-9960.

[32] N. Schmid, A.P. Eichenberger, A. Choutko, S. Riniker, M. Winger, A.E. Mark, W.F. Van Gunsteren, Definition and testing of the GROMOS force-field versions 54A7 and 54B7, European Biophysics Journal, 40 (2011) 843-856. 
[33] G. Sastre, J. Kärger, D.M. Ruthven, Molecular Dynamics Study of Diffusion and Surface Permeation of Benzene in Silicalite, Journal of Physical Chemistry C, 122 (2018) 7217-7225.

[34] T. Darden, D. York, L. Pedersen, Particle mesh Ewald: An N.log(N) method for Ewald sums in large systems.pdf, The Journal of Chemical Physics, 98 (1993) 10089-10092.

[35] C. Perego, M. Salvalaglio, M. Parrinello, Molecular dynamics simulations of solutions at constant chemical potential, The Journal of Chemical Physics, 142 (2015) 144113.

[36] https://github.com/mme-ucl/CmuMD

[37] M. Allen, DJ Tildesley Computer simulation of liquids, in, Clarendon Press, Oxford, 1987.

[38] D. Dubbeldam, S. Calero, D.E. Ellis, R.Q. Snurr, RASPA: Molecular simulation software for adsorption and diffusion in flexible nanoporous materials, Molecular Simulation, 42 (2016) 81-101.

[39] K. Zhang, R.P. Lively, J.D. Noel, M.E. Dose, B.A. McCool, R.R. Chance, W.J. Koros, Adsorption of water and ethanol in MFI-type zeolites, Langmuir, 28 (2012) 8664-8673.

[40] M. Trzpit, M. Soulard, J. Patarin, N. Desbiens, F. Cailliez, A. Boutin, I. Demachy, A.H. Fuchs, The Effect of Local Defects on Water Adsorption in Silicalite-1 Zeolite: A Joint Experimental and Molecular Simulation Study, Langmuir, 23 (2007) 10131-10139.

[41] M. Trzpit, M. Soulard, J. Patarin, Water intrusion in mesoporous silicalite-1: An increase of the stored energy, Microporous and Mesoporous Materials, 117 (2009) 627-634.

[42] A. Martin-Calvo, J.J. Gutierrez-Sevillano, D. Dubbeldam, S. Calero, Using Aliphatic Alcohols to Tune Benzene Adsorption in MAF-6, Advanced Theory and Simulations, 2 (2019) 1900112. 


\title{
Supporting Information
}

for

The Role of Hydrogen Bonding in the Dehydration of Bioalcohols in Hydrophobic Pervaporation Membranes

\author{
Rafael M. Madero-Castro ${ }^{1,2}$, Sofia Calero ${ }^{* 2,3}$ and A. Ozgur Yazaydin*1
}

${ }^{1}$ Department of Chemical Engineering, University College London, London, WC1E 7JE, UK

${ }^{2}$ Department of Physical, Chemical and Natural Systems, Universidad Pablo de Olavide, Ctra. Utrera Km. 1 Seville ES-41013, Spain

${ }^{3}$ Materials Simulation and Modelling, Department of Applied Physics, Eindhoven University of Technology, 5600 MB Eindhoven, The Netherlands.

Corresponding authors:

Email: ozgur.yazaydin@ucl.ac.uk

Email: scalero@upo.es 


\section{Force field parameters for the MFI membrane atoms}

Table S1. Atom types (a) and parameters for the non-bonded Lennard-Jones potential (b), bonds (c), angles (d) and dihedrals.

(a) Atom types, and their corresponding masses and the partial charges

\begin{tabular}{|ccc|}
\hline Atom & Mass[u] & Charge[e] \\
\hline & 65.380 & 2.100 \\
\hline O1 & 15.999 & -0.725 \\
\hline O2 & 15.999 & -1.05 \\
\hline H1 & 1.008 & 0.200 \\
\hline CH3_MeOH & 15.03452 & 0.265 \\
\hline CH3_EOH & 15.03452 & 0 \\
\hline CH2_EOH & 14.02658 & 0.265 \\
\hline O_alc & 15.9994 & -0.7 \\
\hline H_alc & 1.008 & 0.435 \\
\hline O_wat & 15.9994 & -0.8476 \\
\hline H_wat & 1.008 & 0.4238 \\
\hline
\end{tabular}

(b) Non-Bonded potential

$$
V_{i j}=4 \varepsilon_{i j}\left(\left(\frac{\sigma_{i j}}{r_{i j}}\right)^{12}-\left(\frac{\sigma_{i j}}{r_{i j}}\right)^{6}\right)
$$

\begin{tabular}{|cccc|}
\hline $\mathbf{A}_{\mathbf{i}}$ & $\mathrm{A}_{\mathbf{j}}$ & $\boldsymbol{\varepsilon}[\mathrm{kJ} / \mathrm{mol}]$ & $\sigma[\mathrm{nm}]$ \\
\hline $\mathrm{Si}$ & $\mathrm{Si}$ & $6.89057 \cdot 10^{-7}$ & 0.334478 \\
\hline $\mathrm{O} 1$ & $\mathrm{O} 1$ & 0.7986 & 0.3107819 \\
\hline $\mathrm{O} 2$ & $\mathrm{O} 2$ & 0.7986 & 0.3107819 \\
\hline $\mathrm{H} 1$ & $\mathrm{H} 1$ & 0.0 & 0.0 \\
\hline $\mathrm{Si}$ & $\mathrm{O} 1$ & 0.10153396046 & 0.247105204426 \\
\hline $\mathrm{Si}$ & $\mathrm{O} 2$ & 0.00164729035 & 0.341646346144 \\
\hline CH3_MeOH & $\mathrm{CH}$ _MeOH & 0.815 & 0.375 \\
\hline CH3_EOH & CH3_EOH & 0.815 & 0.375 \\
\hline CH2_EOH & CH2_EOH & 0.382 & 0.375 \\
\hline O_alc & O_alc & 0.773 & 0.302 \\
\hline H_alc & H_alc & - & - \\
\hline O_wat & O_wat & 0.650 & 0.3166 \\
\hline H_wat & H_wat & - & - \\
\hline
\end{tabular}


(c) Harmonic Bond Potential

$$
V_{i j}=\frac{1}{2} k_{b}\left(r_{i j}-b_{0}\right)^{2}
$$

\begin{tabular}{|cccc|}
\hline Ai & Aj & $\mathrm{kb}\left[\mathrm{kJ} / \mathrm{mol}^{\circ} \mathrm{nm}^{-2}\right]$ & bo[nm] \\
\hline O1 & H1 & 4640.07 & 0.09476 \\
\hline CH3_MeOH & O_alc & 502416.0 & 0.1430 \\
\hline CH3_EOH & CH2_EOH & 502416.0 & 0.1540 \\
\hline CH2_EOH & O_alc & 502416.0 & 0.1430 \\
\hline O_alc & H_alc & 502416.0 & 0.0945 \\
\hline O_wat & H_wat & 345168.134 & 0.1000 \\
\hline
\end{tabular}

(d) Harmonic Angle potential

$$
V_{i j k}(\theta)=\frac{1}{2} k_{\theta}\left(\theta_{i j k}-\theta_{0}\right)^{2}
$$

\begin{tabular}{|ccccc|}
\hline $\mathbf{A}_{\mathbf{i}}$ & $\mathbf{A}_{\mathbf{j}}$ & $\mathbf{A}_{\mathbf{k}}$ & $\mathrm{ke}\left[\mathrm{kJ} / \mathrm{mol}^{\left.-\mathrm{rad}^{-2}\right]}\right.$ & $\boldsymbol{\Theta}\left(^{\circ}\right)$ \\
\hline $\mathrm{O} 1$ & $\mathrm{Si}$ & $\mathrm{O} 2$ & 144.1876 & 109.47 \\
\hline $\mathrm{O} 2$ & $\mathrm{Si}$ & $\mathrm{O} 2$ & 144.1876 & 109.47 \\
\hline $\mathrm{Si}$ & $\mathrm{O} 2$ & $\mathrm{Si}$ & 149.6391 & 142.71 \\
\hline CH3_MeOH & O_alc & H_alc & 460.61833 & 108.50 \\
\hline CH3_EOH & CH2_EOH & O_alc & 419.04626 & 109.47 \\
\hline CH3_EOH & O_alc & H_alc & 460.61833 & 108.50 \\
\hline H_wat & O_wat & H_wat & 383.18666 & 109.47 \\
\hline
\end{tabular}

(e) Dihedral potential

\begin{tabular}{|c|c|c|c|c|c|c|c|c|c|}
\hline $\mathbf{A}_{\mathbf{i}}$ & $\mathbf{A}_{\mathbf{j}}$ & $\mathbf{A}_{\mathbf{k}}$ & $\mathbf{A l}_{\mathbf{l}}$ & $C_{0} *$ & $C_{1} *$ & $\boldsymbol{C}_{2} *$ & $C_{3} *$ & $C_{4} *$ & $C_{5}^{*}$ \\
\hline CH3_EOH & CH2_EOH & O_alc & H_alc & 2.822 & 2.943 & 0.485 & -6.25 & 0.0 & 0.0 \\
\hline
\end{tabular}

$$
V_{R B}\left(\theta_{i j k l}\right)=\sum_{n=0}^{5} C_{n}(\cos (\psi))^{n}, \quad \text { where } \psi=\theta_{i j k l}-180^{\circ}
$$

\section{S2. GCMC methodology}

To obtain the amount of molecules of alcohol and water inside the structure we performed $2 \cdot 10^{5}$ equilibrate cycles, followed by $10^{6}$ production run cycles in the grand-canonical ensemble (GCMC). In these simulations, we considered a rigid periodic crystal using the crystallographically determined position of the atoms, because the flexibility does not affect so much to adsorption. The MFI unit cell was replicated 2, 2 and 3 times in the $\mathrm{x}, \mathrm{y}$ and $\mathrm{z}$ direction, respectively, to surpass twice the spherical cut-off used for non-bonded interactions; i.e. $12 \AA$. Translation, rotation and insertion/deletion moves were sampled with equal probabilities. 


\section{S3. CGD-MD parameters}

CGD-MD is a simulation method that provides regions in which the particle concentration is maintained at a target value. With this aim, a bi-directional bias force is applied on the particles to keep the concentration of the particles constant in the control regions. The expression for the bi-directional force is

$$
F(z)=k_{i}\left(n_{i}^{C R}-n_{0 i}\right) \cdot G\left(z, Z_{F}\right)
$$

where $k_{i}$ is a force constant, $n_{i}^{C R}$ is the instantaneous density in the designated control regions (CR) while $n_{0 i}$ is the target density. $G\left(z, Z_{F}\right)$ is a bell-shaped function whose value is equal to 1 for $z=Z_{F}$ and it is null outside of a $Z_{F}$-centered $w$ range, i.e. $G\left(z, Z_{F}\right)$ delimits the region where forces are applied. Mathematical expression of this bell-shaped function is

$$
G\left(z, Z_{F}\right)=\frac{1}{4 w}\left[1+\cosh \left(\frac{z-Z_{F}}{w}\right)\right]^{-1}
$$

Instantaneous particle density is given by

$$
n_{i}^{C R}=\frac{1}{V^{C R}} \sum_{j=1}^{N_{i}} \theta\left(z_{j}\right) \quad \text { where } \theta\left(z_{j}\right)= \begin{cases}1 & \text { if } z_{j} \in C R \\ 0 & \text { otherwise }\end{cases}
$$

where $V^{C R}$ the volume of the $\mathrm{CR}$.

Table S2. Force constants and width of the control regions in the inlet and outlet of the membrane

\begin{tabular}{|ccccc|}
\hline Side & $\mathbf{w}[\mathrm{nm}]$ & $\mathbf{k}_{\mathrm{i}}\left[\mathrm{kJ} \cdot \mathrm{nm}^{3} / \mathrm{mol}\right]$ & $\mathbf{Z}_{\mathrm{F}}[\mathrm{nm}]$ & $\mathbf{V}^{\mathrm{CR}}[\mathrm{nm}] /\left(\mathrm{L}_{\mathrm{X}} \mathrm{L}_{\mathrm{y}}\right)$ \\
\hline Inlet & 0.25 & 500 & 4.107 & 2.5 \\
\hline Outlet & 0.25 & 5000 & 24.690 & 2.5 \\
\hline
\end{tabular}




\section{S4. Supplementary results from CGD-MD simulations}

Table S3. Target concentrations and computed average concentrations of methanol, ethanol and water in control regions in single component CGD-MD simulations.

\begin{tabular}{|l|c|c|c|c|}
\hline \multirow{2}{*}{} & \multicolumn{2}{|c|}{ Feed control region (FCR) } & \multicolumn{2}{c|}{ Permeate control region (PCR) } \\
\cline { 2 - 5 } & $\begin{array}{c}\text { Target } \\
\text { concentration } \\
{\left[\text { molecules } / \mathrm{nm}^{3}\right]}\end{array}$ & $\begin{array}{c}\text { Average } \\
\text { concentration } \\
{\left[\text { molecules } / \mathrm{nm}^{3}\right]}\end{array}$ & $\begin{array}{c}\text { Target } \\
\text { concentration } \\
{\left[\text { molecules } / \mathrm{nm}^{3}\right]}\end{array}$ & $\begin{array}{c}\text { Average } \\
\text { concentration } \\
{\left[\text { molecules } / \mathrm{nm}^{3}\right]}\end{array}$ \\
\hline Methanol & 15.23 & $14.99 \pm 0.06$ & 0 & $0.004 \pm 0.007$ \\
\hline Ethanol & 10.31 & $10.16 \pm 0.05$ & 0 & $0.028 \pm 0.007$ \\
\hline Water & 33.46 & $33.7 \pm 0.1$ & 0 & $0^{*}$ \\
\hline
\end{tabular}

* Water does not enter the MFI membrane in the single component simulation.

(a)

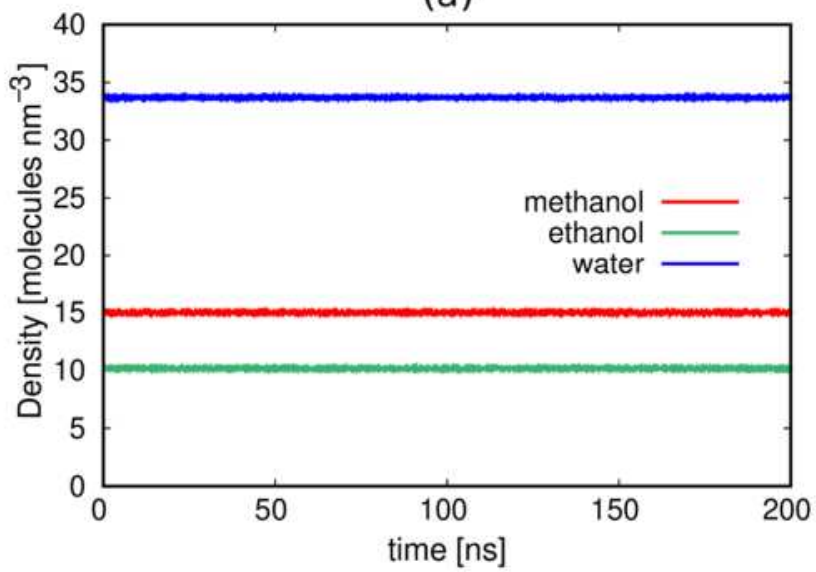

(b)

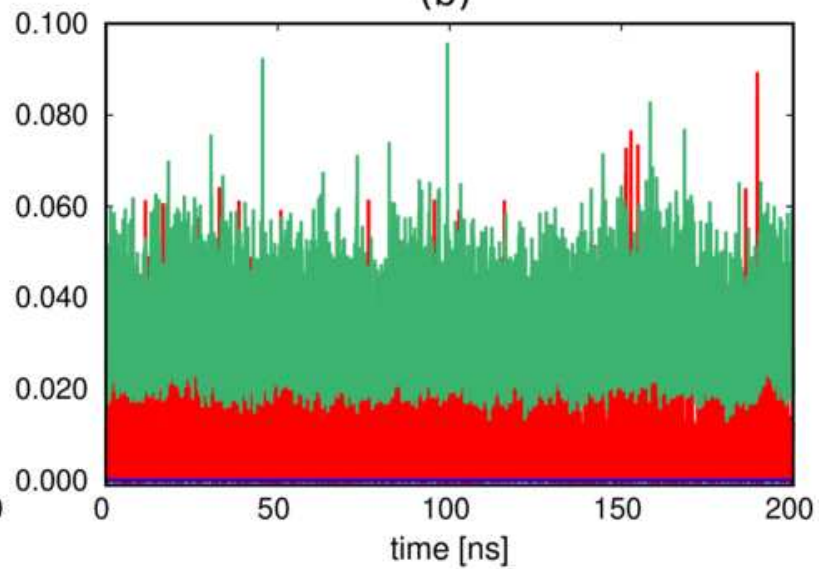

Figure S1. Moving averages of the molecular density (calculated using preceding 100 data points) as a function of simulation time for methanol (red), ethanol (green) and water (blue) in the FCR (a) and PCR (b). Note that because pure water does not permeate through the MFI membrane, water density in PCR is effectively zero. For methanol and ethanol densities in PCR are noisy due to very low statistical probability of finding a particle in vacuum. Concentrations averaged over the $200 \mathrm{~ns}$ production runs are given in Table S3. 
Table S4. Target concentrations and computed average concentrations of methanol, ethanol and water in control regions in alcohol-water mixture CGD-MD simulations.

\begin{tabular}{|c|c|c|c|c|}
\hline & \multicolumn{2}{|c|}{ Feed control region (FCR) } & \multicolumn{2}{|c|}{ Permeate control region (PCR) } \\
\hline & $\begin{array}{c}\text { Target } \\
\text { concentration } \\
{\left[\text { molecules } / \mathrm{nm}^{3}\right]}\end{array}$ & $\begin{array}{c}\text { Average } \\
\text { concentration } \\
{\left[\text { molecules / } \mathrm{nm}^{3} \text { ] }\right.} \\
\text { Alcohol / Water }\end{array}$ & $\begin{array}{c}\text { Target } \\
\text { concentration } \\
{\left[\text { molecules } / \mathrm{nm}^{3}\right]}\end{array}$ & $\begin{array}{c}\text { Average } \\
\text { concentration } \\
\text { [molecules / } \mathrm{nm}^{3} \text { ] } \\
\text { Alcohol / Water }\end{array}$ \\
\hline $\begin{array}{l}\text { Methanol- } \\
\text { Water }\end{array}$ & 10.29 & $\begin{array}{c}10.43 \pm 0.07 \\
/ \\
10.39 \pm 0.09\end{array}$ & 0 & $\begin{array}{c}0.002 \pm 0.004 \\
/ \\
0.0002 \pm 0.0008\end{array}$ \\
\hline Ethanol-Water & 7.88 & $\begin{array}{c}7.92 \pm 0.06 \\
/ \\
7.89 \pm 0.09\end{array}$ & 0 & $\begin{array}{c}0.002 \pm 0.004 \\
/ \\
0.0001 \pm 0.0006\end{array}$ \\
\hline
\end{tabular}




\section{methanol-water}
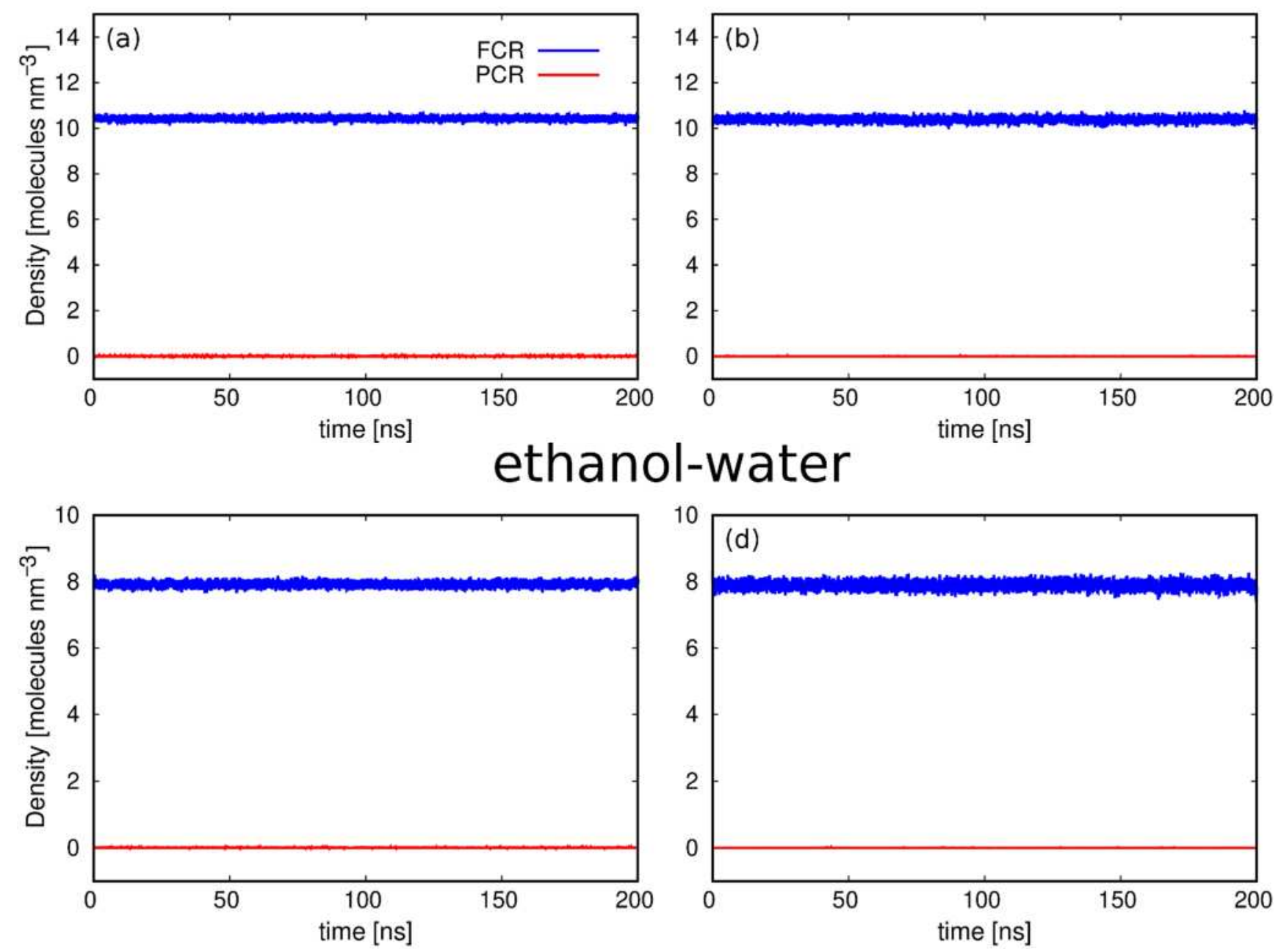

Figure S2. Moving averages of the molecular density (calculated using preceding 100 data points) as a function of simulation time in the FCR (blue) and PCR (red) in the methanol-water (top) and ethanolwater (bottom) mixtures. The data for alcohols are shown on left side (methanol (a) and ethanol (c)) and water on right side ((b) and (d)) Concentrations averaged over the 200 ns production runs are given in Table S4. 